\title{
Poisson Vector Fields on Weil Bundles
}

\author{
Norbert Mahoungou Moukala1, Basile Guy Richard Bossoto ${ }^{1,2}$ \\ ${ }^{1}$ Faculty of Sciences and Technology, Marien NGOUABI University, Brazzaville, Congo \\ ${ }^{2}$ Institut de Recherche en Sciences Exactes et Naturelles (IRSEN), Brazzaville, Congo \\ Email:nmahomouk@yahoo.fr, bossotob@yahoo.fr
}

Received 2 October 2015; accepted 6 November 2015; published 9 November 2015

Copyright $(\subset 2015$ by authors and Scientific Research Publishing Inc.

This work is licensed under the Creative Commons Attribution International License (CC BY).

http://creativecommons.org/licenses/by/4.0/

(c) (i) Open Access

\begin{abstract}
In this paper, $M$ is a smooth manifold of finite dimension $n, A$ is a local algebra and $M^{A}$ is the associated Weil bundle. We study Poisson vector fields on $M^{A}$ and we prove that all globally hamiltonian vector fields on $M^{A}$ are Poisson vector fields.
\end{abstract}

\section{Keywords}

Weil Algebra, Weil Bundle, Poisson Manifold, Lie Derivative, Poisson 2-Form

\section{Introduction}

A Weil algebra or local algebra (in the sense of André Weil) [1], is a finite dimensional, associative, commutative and unitary algebra $A$ over $\mathbb{R}$ in which there exists a unique maximum ideal $\mathfrak{m}$ of codimension 1 . In his case, the factor space $A / \mathfrak{m}$ is one-dimensional and is identified with the algebra of real numbers $\mathbb{R}$. Thus $A=\mathbb{R} \oplus \mathfrak{m}$ and $\mathbb{R}$ is identified with $\mathbb{R} \cdot 1_{A}$, where $1_{A}$ is the unit of $A$.

In what follows we denote by $A$ a Weil algebra, $M$ a smooth manifold, $C^{\infty}(M)$ the algebra of smooth functions on $M$.

A near point of $x \in M$ of kind $A$ is a homomorphism of algebras

$$
\xi: C^{\infty}(M) \rightarrow A
$$

such that for any $f \in C^{\infty}(M),[\xi(f)-f(x)] \in \mathfrak{m}$.

We denote by $M_{x}^{A}$ the set of near points of $x$ of kind $A$ and $M^{A}=\bigcup_{x \in M} M_{x}^{A}$ the set of near points on $M$ of kind $A$. The set $M^{A}$ is a smooth manifold of dimension $\operatorname{dim} M \times \operatorname{dim} A$ and called manifold of infinitely near points on $M$ of kind $A$ [1]-[3], or simply the Weil bundle [4] [5].

If $f: M \rightarrow \mathbb{R}$ is a smooth function, then the map

$$
f^{A}: M^{A} \rightarrow \mathbb{R}^{A}=A, \xi \mapsto\left[f^{A}(\xi)\right]\left(i d_{\mathbb{R}}\right)=\xi\left(i d_{\mathbb{R}} \circ f\right)=\xi(f)
$$


is differentiable of class $C^{\infty}$ [4] [6]. The set, $C^{\infty}\left(M^{A}, A\right)$ of smooth functions on $M^{A}$ with values on $A$, is a commutative algebra over $A$ with unit and the map

$$
C^{\infty}(M) \rightarrow C^{\infty}\left(M^{A}, A\right), f \mapsto f^{A}
$$

is an injective homomorphism of algebras. Then, we have:

$$
(f+g)^{A}=f^{A}+g^{A} ;(\lambda \cdot f)^{A}=\lambda \cdot f^{A} ;(f \cdot g)^{A}=f^{A} \cdot g^{A} .
$$

We denote $\mathfrak{X}\left(M^{A}\right)$, the set of vector fields on $M^{A}$ and $\operatorname{Der}_{A}\left[C^{\infty}\left(M^{A}, A\right)\right]$ the set of $A$-linear maps

$$
X: C^{\infty}\left(M^{A}, A\right) \rightarrow C^{\infty}\left(M^{A}, A\right)
$$

such that

$$
X(\varphi \cdot \psi)=X(\varphi) \cdot \psi+\varphi \cdot X(\psi), \text { for any } \varphi, \psi \in C^{\infty}\left(M^{A}, A\right)
$$

Thus [4],

$$
\mathfrak{X}\left(M^{A}\right)=\operatorname{Der}_{A}\left[C^{\infty}\left(M^{A}, A\right)\right]
$$

If

$$
\theta: C^{\infty}(M) \rightarrow C^{\infty}(M)
$$

is a vector field on $M$, then there exists one and only one $A$-linear derivation

$$
\theta^{A}: C^{\infty}\left(M^{A}, A\right) \rightarrow C^{\infty}\left(M^{A}, A\right)
$$

called prolongation of the vector field $\theta$ [4] [6], such that

$$
\theta^{A}\left(f^{A}\right)=[\theta(f)]^{A}, \text { for any } f \in C^{\infty}(M) .
$$

Let $\Omega_{\mathbb{R}}\left[C^{\infty}(M)\right]$ be the $C^{\infty}(M)$-module of Kälher differentials of $C^{\infty}(M)$ and

$$
\delta_{M}: C^{\infty}(M) \rightarrow \Omega_{\mathbb{R}}\left[C^{\infty}(M)\right], f \mapsto \overline{f \otimes 1_{C^{\infty}(M)}-1_{C^{\infty}(M)} \otimes f}
$$

the canonical derivation which the image of $\delta_{M}$ generates the $C^{\infty}(M)$-module $\Omega_{\mathbb{R}}\left[C^{\infty}(M)\right]$ i.e. for $x \in \Omega_{\mathbb{R}}\left[C^{\infty}(M)\right]$,

$$
x=\sum_{i \in I: \text { finite }} f_{i} \cdot \delta_{M}\left(g_{i}\right),
$$

with $f_{i}, g_{i} \in C^{\infty}(M)$ for any $i \in I$ [7] et [8].

We denote $\Omega_{A}\left[C^{\infty}\left(M^{A}, A\right)\right]$, the $C^{\infty}\left(M^{A}, A\right)$-module of Kälher differentials of $C^{\infty}\left(M^{A}, A\right)$ which are $A$-linear. In this case, for $\varphi \in C^{\infty}\left(M^{A}, A\right)$, we denote $\overline{\varphi \otimes 1_{C^{\infty}\left(M^{A}, A\right)}-1_{C^{\infty}\left(M^{A}, A\right)} \otimes \varphi}$, the class of $\varphi \otimes 1_{C^{\infty}\left(M^{A}, A\right)}-1_{C^{\infty}\left(M^{A}, A\right)} \otimes \varphi$ in $C^{\infty}\left(M^{A}, A\right)$.

The map

$$
C^{\infty}(M) \rightarrow \Omega_{A}\left[C^{\infty}\left(M^{A}, A\right)\right], f \mapsto\left[\delta_{M}(f)\right]^{A}=\overline{f^{A} \otimes 1_{C^{\infty}\left(M^{A}, A\right)}-1_{C^{\infty}\left(M^{A}, A\right)} \otimes f^{A}}
$$

is a derivation and there exists a unique $A$-linear derivation

$$
\delta_{M^{A}}^{A}: C^{\infty}\left(M^{A}, A\right) \rightarrow \Omega_{A}\left[C^{\infty}\left(M^{A}, A\right)\right]
$$

such that

$$
\delta_{M^{A}}^{A}\left(f^{A}\right)=\left[\delta_{M}(f)\right]^{A}
$$

for any $f \in C^{\infty}(M)$ [9]. Moreover the map 


$$
\Omega_{\mathbb{R}}\left[C^{\infty}(M)\right] \rightarrow \Omega_{A}\left[C^{\infty}\left(M^{A}, A\right)\right], x \mapsto x^{A}
$$

is an injective homomorphism of $\mathbb{R}$-modules. Thus, the pair $\left(\Omega_{A}\left[C^{\infty}\left(M^{A}, A\right)\right], \delta_{M^{A}}^{A}\right)$ satisfies the following universal property: for every $C^{\infty}\left(M^{A}, A\right)$-module $E$ and every $A$-derivation

$$
\Phi: C^{\infty}\left(M^{A}, A\right) \rightarrow E
$$

there exists a unique $C^{\infty}\left(M^{A}, A\right)$-linear map

$$
\tilde{\Phi}: \Omega_{A}\left[C^{\infty}\left(M^{A}, A\right)\right] \rightarrow E
$$

such that

$$
\tilde{\Phi} \circ \delta_{M^{A}}^{A}=\Phi
$$

In other words, there exists a unique $\tilde{\Phi}$ which makes the following diagram commutative

$$
\begin{array}{cc}
\Omega_{A}\left[C^{\infty}\left(M^{A}, A\right)\right] & \\
\delta_{M^{A}}^{A} \uparrow & \searrow \\
C^{\infty}\left(M^{A}, A\right) & \underset{\Phi}{\tilde{\Phi}} E
\end{array}
$$

This fact implies the existence of a natural isomorphism of $C^{\infty}\left(M^{A}, A\right)$-modules

$$
\operatorname{Hom}_{C^{\infty}\left(M^{A}, A\right)}\left(\Omega_{A}\left[C^{\infty}\left(M^{A}, A\right)\right], E\right) \rightarrow \operatorname{Der}_{A}\left[C^{\infty}\left(M^{A}, A\right), E\right], \psi \mapsto \psi \circ \delta_{M^{A}}^{A} .
$$

In particular, if $E=C^{\infty}\left(M^{A}, A\right)$, we have

$$
\Omega_{A}\left[C^{\infty}\left(M^{A}, A\right)\right]^{*} \simeq \operatorname{Der}_{A}\left[C^{\infty}\left(M^{A}, A\right)\right]=\mathfrak{X}\left(M^{A}\right) .
$$

For any $p \in \mathbb{N}, \Lambda^{p}\left(\Omega_{A}\left[C^{\infty}\left(M^{A}, A\right)\right]\right)=\mathfrak{L}_{\text {sks }}^{p}\left(\Omega_{A}\left[C^{\infty}\left(M^{A}, A\right)\right], C^{\infty}\left(M^{A}, A\right)\right)$ denotes the $C^{\infty}\left(M^{A}, A\right)$ module of skew-symmetric multilinear forms of degree $p$ from $\Omega_{A}\left[C^{\infty}\left(M^{A}, A\right)\right]$ into $C^{\infty}\left(M^{A}, A\right)$ and

$$
\Lambda\left(\Omega_{A}\left[C^{\infty}\left(M^{A}, A\right)\right]\right)=\bigoplus_{p \in \mathbb{N}} \Lambda^{p}\left(\Omega_{A}\left[C^{\infty}\left(M^{A}, A\right)\right]\right)
$$

the exterior $C^{\infty}\left(M^{A}, A\right)$-algebra of $\Omega_{A}\left[C^{\infty}\left(M^{A}, A\right)\right]$ called algebra of Kähler forms on $C^{\infty}\left(M^{A}, A\right)$.

$$
\begin{gathered}
\Lambda^{0}\left(\Omega_{A}\left[C^{\infty}\left(M^{A}, A\right)\right]\right)=C^{\infty}\left(M^{A}, A\right), \\
\Lambda^{1}\left(\Omega_{A}\left[C^{\infty}\left(M^{A}, A\right)\right]\right)=\Omega_{A}\left[C^{\infty}\left(M^{A}, A\right)\right]^{*} .
\end{gathered}
$$

If $\eta \in \Lambda^{p}\left(\Omega_{\mathbb{R}}\left[C^{\infty}(M)\right]\right)$, then $\eta$ is of the form $\delta_{M}\left(f_{1}\right) \wedge \cdots \wedge \delta_{M}\left(f_{p}\right)$ with $f_{1}, f_{2}, \cdots, f_{p} \in C^{\infty}(M)$. Thus, the $C^{\infty}\left(M^{A}, A\right)$-module $\Lambda^{p}\left(\Omega_{A}\left[C^{\infty}\left(M^{A}, A\right)\right]\right)$ is generated by elements of the form

$$
\eta^{A}=\delta_{M^{A}}^{A}\left(\varphi_{1}\right) \wedge \cdots \wedge \delta_{M^{A}}^{A}\left(\varphi_{p}\right)
$$

with $\varphi_{1}=f_{1}^{A}, \cdots, \varphi_{p}=f_{p}^{A} \in C^{\infty}\left(M^{A}, A\right)$.

Let

$$
\sigma_{\theta}:\left[\Omega_{\mathbb{R}}\left[C^{\infty}(M)\right]\right]^{p} \rightarrow \Lambda^{p}\left(\Omega_{\mathbb{R}}\left[C^{\infty}(M)\right]\right),
$$

be the $C^{\infty}(M)$-skew-symmetric multilinear map such that 


$$
\sigma_{\theta}\left(x_{1}, x_{2}, \cdots, x_{p}\right)=\sum_{i=1}^{p}(-1)^{i-1} \tilde{\theta}\left(x_{i}\right) \cdot x_{1} \wedge \cdots \wedge \hat{x}_{i} \wedge \cdots \wedge x_{p},
$$

for any $x_{1}, x_{2}, \cdots, x_{p} \in \Omega_{\mathbb{R}}\left[C^{\infty}(M)\right]$ and, where

$$
\tilde{\theta}: \Omega_{\mathbb{R}}\left[C^{\infty}(M)\right] \rightarrow C^{\infty}(M)
$$

is a unique $C^{\infty}(M)$-linear map such that $\tilde{\theta} \circ \delta_{M}=\theta$ [8]. Then,

$$
\sigma_{\theta^{A}}^{A}:\left[\Omega_{A}\left[C^{\infty}\left(M^{A}, A\right)\right]\right]^{p} \rightarrow \Lambda^{p}\left(\Omega_{A}\left[C^{\infty}\left(M^{A}, A\right)\right]\right)
$$

is a unique $C^{\infty}(M)$-skew-symmetric multilinear map such that

$$
\sigma_{\theta^{A}}^{A}\left(x_{1}^{A}, x_{2}^{A}, \cdots, x_{p}^{A}\right)=\left[\sigma_{\theta}\left(x_{1}, x_{2}, \cdots, x_{p}\right)\right]^{A} .
$$

We denote

$$
\widetilde{\sigma_{\theta^{A}}^{A}}: \Lambda^{p}\left(\Omega_{A}\left[C^{\infty}\left(M^{A}, A\right)\right]\right) \rightarrow \Lambda^{p-1}\left(\Omega_{A}\left[C^{\infty}\left(M^{A}, A\right)\right]\right),
$$

the unique $C^{\infty}\left(M^{A}, A\right)$-skew-symmetric multilinear map such that

$$
\widetilde{\sigma_{\theta^{A}}^{A}}\left(x_{1}^{A} \wedge x_{2}^{A} \wedge \cdots \wedge x_{p}^{A}\right)=\sigma_{\theta^{A}}^{A}\left(x_{1}^{A}, x_{2}^{A}, \cdots, x_{p}^{A}\right)
$$

i.e. $\sigma_{\theta^{A}}^{A}$ induces a derivation

$$
i_{\theta^{A}}=\widetilde{\sigma_{\theta^{A}}^{A}}: \Lambda\left(\Omega_{A}\left[C^{\infty}\left(M^{A}, A\right)\right]\right) \rightarrow \Lambda\left(\Omega_{A}\left[C^{\infty}\left(M^{A}, A\right)\right]\right)
$$

of degree -1 [9].

We recall that a Poisson structure on a smooth manifold $M$ is due to the existence of a bracket $\{,\}_{M}$ on $C^{\infty}(M)$ such that the pair $\left(C^{\infty}(M),\{,\}_{M}\right)$ is a real Lie algebra such that, for any $f \in C^{\infty}(M)$ the map

$$
\operatorname{ad}(f): C^{\infty}(M) \rightarrow C^{\infty}(M), g \mapsto\{f, g\}_{M}
$$

is a derivation of commutative algebra i.e.

$$
\{f, g \cdot h\}_{M}=\{f, g\}_{M} \cdot h+g \cdot\{f, h\}_{M}
$$

for $f, g, h \in C^{\infty}(M)$. In this case we say that $C^{\infty}(M)$ is a Poisson algebra and $M$ is a Poisson manifold [10] [11].

The manifold $M$ is a Poisson manifold if and only if there exists a skew-symmetric 2-form

$$
\omega_{M}: \Omega_{\mathbb{R}}\left[C^{\infty}(M)\right] \times \Omega_{\mathbb{R}}\left[C^{\infty}(M)\right] \rightarrow C^{\infty}(M)
$$

such that for any $f$ and $g$ in $C^{\infty}(M)$,

$$
\{f, g\}_{M}=-\omega_{M}\left[\delta_{M}(f), \delta_{M}(g)\right]
$$

defines a structure of Lie algebra over $C^{\infty}(M)$ [8]. In this case, we say that $\omega_{M}$ is the Poisson 2-form of the Poisson manifold $M$ and we denote $\left(M, \omega_{M}\right)$ the Poisson manifold of Poisson 2-form $\omega_{M}$.

\section{Poisson 2-Form on Weil Bundles}

When $\left(M,\{,\}_{M}\right)$ is a Poisson manifold, the map

$$
\text { ad }: C^{\infty}(M) \rightarrow \operatorname{Der}_{\mathbb{R}}\left[C^{\infty}(M)\right], f \mapsto \operatorname{ad}(f)
$$

such that $[\operatorname{ad}(f)](g)=\{f, g\}_{M}$ for any $g \in C^{\infty}(M)$, is a derivation. Thus, there exists a derivation

$$
\operatorname{ad}^{A}: C^{\infty}\left(M^{A}, A\right) \rightarrow \operatorname{Der}_{A}\left[C^{\infty}\left(M^{A}, A\right)\right]
$$


such that

$$
\operatorname{ad}^{A}\left(f^{A}\right)=[\operatorname{ad}(f)]^{A}
$$

Let

$$
\widetilde{a d^{A}}: \Omega_{A}\left[C^{\infty}\left(M^{A}, A\right)\right] \rightarrow \operatorname{Der}_{A}\left[C^{\infty}\left(M^{A}, A\right)\right]
$$

be a unique $C^{\infty}\left(M^{A}, A\right)$-linear map such that

$$
\widetilde{a d^{A}} \circ \delta_{M^{A}}^{A}=a d^{A} .
$$

Let us consider the canonical isomorphism

$$
\sigma_{M^{A}}: \Omega_{A}\left[C^{\infty}\left(M^{A}, A\right)\right]^{*} \rightarrow \operatorname{Der}_{A}\left[C^{\infty}\left(M^{A}, A\right)\right], \Psi \mapsto \Psi \circ \delta_{M^{A}}^{A}
$$

and let

$$
\sigma_{M^{A}}^{-1} \circ \widetilde{a d^{A}}: \Omega_{A}\left[C^{\infty}\left(M^{A}, A\right)\right]^{\widetilde{a d^{A}}} \rightarrow \operatorname{Der}_{A}\left[C^{\infty}\left(M^{A}, A\right)\right]^{\sigma_{M^{A}}^{-1}} \Omega_{A}\left[C^{\infty}\left(M^{A}, A\right)\right]^{*}
$$

be the map.

Proposition 1. [9] If $\left(M, \omega_{M}\right)$ is a Poisson manifold, then the map,

$$
\omega_{M^{A}}^{A}: \Omega_{A}\left[C^{\infty}\left(M^{A}, A\right)\right] \times \Omega_{A}\left[C^{\infty}\left(M^{A}, A\right)\right] \rightarrow C^{\infty}\left(M^{A}, A\right)
$$

such that for any $X, Y \in \Omega_{A}\left[C^{\infty}\left(M^{A}, A\right)\right]$

$$
\omega_{M^{A}}^{A}(X, Y)=-\left[\sigma_{M^{A}}^{-1} \circ \widetilde{a d^{A}}(X)\right](Y)
$$

is a skew-symmetric 2-form on $\Omega_{A}\left[C^{\infty}\left(M^{A}, A\right)\right]$ such that

$$
\omega_{M^{A}}^{A}\left(x^{A}, y^{A}\right)=\left[\omega_{M}(x, y)\right]^{A},
$$

for any $x$ and $y$ in $\Omega_{\mathbb{R}}\left[C^{\infty}(M)\right]$. Moreover, $\left(M^{A}, \omega_{M^{A}}^{A}\right)$ is a Poisson manifold.

Theorem 2. [9] The manifold $M^{A}$ is a Poisson manifold if and only if there exists a skew-symmetric 2-form

$$
\omega_{M^{A}}^{A}: \Omega_{A}\left[C^{\infty}\left(M^{A}, A\right)\right] \times \Omega_{A}\left[C^{\infty}\left(M^{A}, A\right)\right] \rightarrow C^{\infty}\left(M^{A}, A\right)
$$

such that for any $\varphi$ and $\psi$ in $C^{\infty}\left(M^{A}, A\right)$,

$$
\{\varphi, \psi\}_{M^{A}}=-\omega_{M^{A}}^{A}\left(\delta_{M^{A}}^{A}(\varphi), \delta_{M^{A}}^{A}(\psi)\right)
$$

defines a structure of A-Lie algebra over $C^{\infty}\left(M^{A}, A\right)$. Moreover, for any fand $g$ in $C^{\infty}(M)$,

$$
\left\{f^{A}, g^{A}\right\}_{M^{A}}=\{f, g\}_{M}^{A} \text {. }
$$

In this case, we will say that $\omega_{M^{A}}^{A}$ is the Poisson 2-form of the A-Poisson manifold $M^{A}$ and we denote $\left(M^{A}, \omega_{M^{A}}^{A}\right)$ the A-Poisson manifold of Poisson 2-form $\omega_{M^{A}}^{A}[$ [9].

\section{Poisson Vector Field on Weil Bundles}

Proposition 3. For any $\theta \in \operatorname{Der}_{\mathbb{R}}\left[C^{\infty}(M)\right]$ and for any $\eta \in \Lambda^{p}\left(\Omega_{\mathbb{R}}\left[C^{\infty}(M)\right]\right)$, we have

$$
i_{\theta^{A}}\left(\eta^{A}\right)=\left[i_{\theta}(\eta)\right]^{A} \text {. }
$$

Proof. If $\eta \in \Lambda^{p}\left(\Omega_{\mathbb{R}}\left[C^{\infty}(M)\right]\right)$, then there exists $f_{1}, f_{2}, \cdots, f_{p} \in C^{\infty}(M)$, such that $\eta=\delta_{M}\left(f_{1}\right) \wedge \cdots \wedge \delta_{M}\left(f_{p}\right)$. Thus, 


$$
\begin{aligned}
i_{\theta^{A}}\left(\eta^{A}\right) & =i_{\theta^{A}}\left(\left[\delta_{M}\left(f_{1}\right) \wedge \cdots \wedge \delta_{M}\left(f_{p}\right)\right]^{A}\right) \\
& =i_{\theta^{A}}\left(\left[\delta_{M}\left(f_{1}\right)\right]^{A} \wedge \cdots \wedge\left[\delta_{M}\left(f_{p}\right)\right]^{A}\right) \\
& =\sigma_{\theta^{A}}^{A}\left(\left[\delta_{M}\left(f_{1}\right)\right]^{A}, \cdots,\left[\delta_{M}\left(f_{p}\right)\right]^{A}\right) \\
& =\left[\sigma_{\theta}\left(\delta_{M}\left(f_{1}\right), \cdots, \delta_{M}\left(f_{p}\right)\right)\right]^{A} \\
& =\left[i_{\theta}\left(\delta_{M}\left(f_{1}\right) \wedge \cdots \wedge \delta_{M}\left(f_{p}\right)\right)\right]^{A} \\
& =\left[i_{\theta}(\eta)\right]^{A} .
\end{aligned}
$$

\subsection{Lie Derivative}

The Lie derivative with respect to $D \in \operatorname{Der}_{A}\left[C^{\infty}\left(M^{A}, A\right)\right]$ is the derivation of degree 0

$$
\mathfrak{L}_{D}=i_{D} \circ \delta_{M^{A}}^{A}+\delta_{M^{A}}^{A} \circ i_{D}: \Lambda\left(\Omega_{A}\left[C^{\infty}\left(M^{A}, A\right)\right]\right) \rightarrow \Lambda\left(\Omega_{A}\left[C^{\infty}\left(M^{A}, A\right)\right]\right) .
$$

Proposition 4. For any $\theta \in \mathfrak{X}(M)$, lthe map

$$
\mathfrak{L}_{\theta^{A}}: \Lambda\left(\Omega_{A}\left[C^{\infty}\left(M^{A}, A\right)\right]\right) \rightarrow \Lambda\left(\Omega_{A}\left[C^{\infty}\left(M^{A}, A\right)\right]\right)
$$

is a unique A-linear derivation such that

$$
\mathfrak{L}_{\theta^{A}}\left(\eta^{A}\right)=\left[\mathfrak{L}_{\theta}(\eta)\right]^{A}
$$

for any $\eta \in \Lambda\left(\Omega_{\mathbb{R}}\left[C^{\infty}(M)\right]\right)$.

Proof. For any $\eta \in \Lambda\left(\Omega_{\mathbb{R}}\left[C^{\infty}(M)\right]\right)$, we have

$$
\begin{aligned}
\mathfrak{L}_{\theta^{A}}\left(\eta^{A}\right) & =i_{\theta^{A}}\left[\delta_{M^{A}}^{A}\left(\eta^{A}\right)\right]+\delta_{M^{A}}^{A}\left[i_{\theta^{A}}\left(\eta^{A}\right)\right] \\
& =i_{\theta^{A}}\left(\left[\delta_{M}(\eta)\right]^{A}\right)+\delta_{M^{A}}^{A}\left(\left[i_{\theta}(\eta)\right]^{A}\right) \\
& =\left(i_{\theta}\left[\delta_{M}(\eta)\right]\right)^{A}+\left(\delta_{M}\left[i_{\theta}(\eta)\right]\right)^{A} \\
& =\left(i_{\theta}\left[\delta_{M}(\eta)\right]+\delta_{M}\left[i_{\theta}(\eta)\right]\right)^{A} \\
& =\left[\mathfrak{L}_{\theta}(\eta)\right]^{A} .
\end{aligned}
$$

A vector field $\theta$ on a Poisson manifold $\left(M, \omega_{M}\right)$ is called Poisson vector field if the Lie derivative of $\omega_{M}$ with respect to $\theta$ vanishes i.e. $\mathfrak{L}_{\theta} \omega_{M}=0$. A vector field

$$
X: C^{\infty}\left(M^{A}, A\right) \rightarrow C^{\infty}\left(M^{A}, A\right)
$$

on a A-Poisson manifold of Poisson 2-form $\omega_{M^{A}}^{A}$ will be said Poisson vector field if $\mathfrak{L}_{X} \omega_{M^{A}}=0$.

Proposition 5. If $\left(M, \omega_{M}\right)$ is a Poisson manifold, then a vector field

is a Poisson vector field if and only if

$$
\theta: C^{\infty}(M) \rightarrow C^{\infty}(M)
$$

$$
\theta^{A}: C^{\infty}\left(M^{A}, A\right) \rightarrow C^{\infty}\left(M^{A}, A\right)
$$

is a Poisson vector field.

Proof. indeed, for any $\theta \in \mathfrak{X}(M)$,

$$
\mathfrak{L}_{\theta^{A}} \omega_{M^{A}}=\left[\mathfrak{L}_{\theta} \omega_{M}\right]^{A} .
$$


Thus, $\mathfrak{L}_{\theta} \omega_{M}=0$ if and only if $\mathfrak{L}_{\theta^{A}} \omega_{M^{A}}=0$.

Proposition 6. Let $\left(M^{A}, \omega_{M^{A}}\right)$ be a A-Poisson manifold. Then, all globally hamiltonian vector fields are Poisson vector fields.

Proof. Let $X$ be a globally hamiltonian vector field, then there exists $\varphi \in C^{\infty}\left(M^{A}, A\right)$ such that $X=a d(\varphi)$ i.e. $X$ is the interior derivation of the Poisson A-algebra $C^{\infty}\left(M^{A}, A\right)$ [6]. For any $\psi$ and $\phi \in C^{\infty}\left(M^{A}, A\right)$,

$$
\begin{aligned}
& \mathfrak{L}_{X} \omega_{M^{A}}\left(\left[\delta_{M^{A}}^{A}(\psi), \delta_{M^{A}}^{A}(\phi)\right]\right)=\left(\mathfrak{L}_{a d(\varphi)} \omega_{M^{A}}\right)\left(\left[\delta_{M^{A}}^{A}(\psi), \delta_{M^{A}}^{A}(\phi)\right]\right) \\
&=\operatorname{ad}(\varphi)\left(\omega_{M^{A}}\left[\delta_{M^{A}}^{A}(\psi), \delta_{M^{A}}^{A}(\phi)\right]\right)-\left(\omega_{M^{A}}\left[\mathfrak{L}_{a d(\varphi)} \delta_{M^{A}}^{A}(\psi), \delta_{M^{A}}^{A}(\phi)\right]\right) \\
&-\left(\omega_{M^{A}}\left[\delta_{M^{A}}^{A}(\psi), \mathfrak{L}_{a d(\varphi)} \delta_{M^{A}}^{A}(\phi)\right]\right) \\
&=-a d(\varphi)(\{\psi, \phi\})-\omega_{M^{A}}\left[\delta_{M^{A}}^{A}\{\varphi, \psi\}, \delta_{M}(\phi)\right]-\omega_{M^{A}}\left[\delta_{M^{A}}^{A}(\psi), \delta_{M^{A}}^{A}\{\varphi, \phi\}\right] \\
&=-\{\varphi,\{\psi, \phi\}\}+\{\{\varphi, \psi\}, \phi\}+\{\psi,\{\varphi, \phi\}\}=-(\{\varphi,\{\psi, \phi\}\}+\{\phi,\{\varphi, \psi\}\}+\{\psi,\{\phi, \varphi\}\})=0 .
\end{aligned}
$$

Thus, all globally hamiltonian vector fields are Poisson vector fields.

When $(M, \Omega)$ is a symplectic manifold, then $\left(M^{A}, \Omega^{A}\right)$ is a symplectic $A$-manifold [6] [12]. For $\varphi \in C^{\infty}\left(M^{A}, A\right)$, we denote $X_{\varphi}$ the unique vector field on $M^{A}$, considered as a derivation of $C^{\infty}\left(M^{A}, A\right)$ into $C^{\infty}\left(M^{A}, A\right)$, such that

$$
i_{X_{\varphi}} \Omega^{A}=d^{A}(\varphi)
$$

where

$$
d^{A}: \Lambda\left(M^{A}, A\right) \rightarrow \Lambda\left(M^{A}, A\right)
$$

denotes the operator of cohomology associated with the representation

$$
\mathfrak{X}\left(M^{A}\right) \rightarrow \operatorname{Der}_{A}\left[C^{\infty}\left(M^{A}, A\right)\right], X \mapsto X .
$$

When $\left(M^{A}, \Omega^{A}\right)$ is a symplectic $A$-manifold, then for any $X \in \mathfrak{X}\left(M^{A}\right)$,

$$
\mathfrak{L}_{X} \Omega^{A}=d^{A}\left(i_{X} \Omega^{A}\right) .
$$

Therefore, all globally hamiltonian vector fields are Poisson vector fields.

Proposition 7. For any $\varphi \in C^{\infty}\left(M^{A}, A\right)$ and for any Poisson vector field $Y$, we have

$$
\left[Y, X_{\varphi}\right]=X_{Y(\varphi)} \text {. }
$$

Proof.

Thus,

$$
\begin{aligned}
i_{\left[Y, X_{\varphi}\right]} \Omega^{A} & =\left[\mathfrak{L}_{Y}, i_{X_{\varphi}}\right] \Omega^{A}=\mathfrak{L}_{Y}\left(i_{X_{\varphi}} \Omega^{A}\right)-i_{X_{\varphi}}\left(\mathfrak{L}_{Y} \Omega^{A}\right) \\
& =\mathfrak{L}_{Y}\left(i_{X_{\varphi}} \Omega^{A}\right)=i_{Y}\left[d^{A}\left(d^{A} \varphi\right)\right]+d^{A}\left[i_{Y}\left(d^{A} \varphi\right)\right] \\
& =d^{A}\left[i_{Y}\left(d^{A} \varphi\right)\right]=d^{A}[Y(\varphi)]=i_{X_{Y(\varphi)}} \Omega^{A} .
\end{aligned}
$$

$$
\left[Y, X_{\varphi}\right]=X_{Y(\varphi)}
$$

\subsection{Example}

When $\alpha=\sum_{i=1}^{n} p_{i} d x_{i}$ is a Liouville form, where $\left(x_{1}, \cdots, x_{n}, p_{1}, \cdots, p_{n}\right)$ is a local system of coordinates in the cotangent bundle $T^{*} M$ of $M$, then $\left(T^{*} M, \Omega=d \alpha=\sum_{i=1}^{n} d p_{i} \Lambda d x_{i}\right)$ is a symplectic manifold on $T^{*} M$ [7]. Let 
$\alpha^{A}$ be the unique differential $A$-form of degree -1 on $T^{*} M^{A}$ such that

$$
d^{A}\left(\alpha^{A}\right)=[d(\alpha)]^{A} .
$$

Thus,

$$
\Omega^{A}=[d(\alpha)]^{A}=d^{A}\left(\alpha^{A}\right)=\sum_{i=1}^{n} d^{A}\left(p_{i}^{A}\right) \Lambda d^{A}\left(x_{i}^{A}\right) .
$$

Therefore, $\left(T^{*} M^{A}, \Omega^{A}=d^{A}\left(\alpha^{A}\right)\right)$ is a symplectic $A$-manifold.

For $H \in C^{\infty}\left(M^{A}, A\right)$, let $X_{H}$ be the globally hamiltonian vector field

$$
i_{X_{H}} \Omega^{A}=-d^{A}(H) .
$$

As [13]

$$
\frac{\partial}{\partial x_{i}^{A}}=\left(\frac{\partial}{\partial x_{i}}\right)^{A},
$$

we have

$$
\begin{gathered}
X_{H}=\sum_{i=1}^{n} f_{i} \cdot \frac{\partial}{\partial x_{i}^{A}}+\sum_{i=1}^{n} g_{i} \cdot \frac{\partial}{\partial p_{i}^{A}}, \\
i_{X_{H}} \Omega^{A}=\sum_{i=1}^{n}\left(i_{X_{H}} \Omega^{A}\right)\left(\frac{\partial}{\partial x_{i}^{A}}\right) d^{A}\left(x_{i}^{A}\right)+\sum_{i=1}^{n}\left(i_{X_{H}} \Omega^{A}\right)\left(\frac{\partial}{\partial p_{i}^{A}}\right) d^{A}\left(p_{i}^{A}\right) \\
=\sum_{i=1}^{n} \Omega^{A}\left(X_{H}, \frac{\partial}{\partial x_{i}^{A}}\right) d^{A}\left(x_{i}^{A}\right)+\sum_{i=1}^{n} \Omega^{A}\left(X_{H}, \frac{\partial}{\partial p_{i}^{A}}\right) d^{A}\left(p_{i}^{A}\right) .
\end{gathered}
$$

As

$$
\begin{aligned}
\Omega^{A}\left(X_{H}, \frac{\partial}{\partial x_{i}^{A}}\right) & =\sum_{j=1}^{n} f_{i} \cdot \Omega^{A}\left(\frac{\partial}{\partial x_{j}^{A}}, \frac{\partial}{\partial x_{i}^{A}}\right)+\sum_{j=1}^{n} g_{j} \cdot \Omega^{A}\left(\frac{\partial}{\partial p_{j}^{A}}, \frac{\partial}{\partial x_{i}^{A}}\right) \\
& =\sum_{j, k}^{n} f_{j} \cdot\left(d^{A}\left(p_{k}^{A}\right) \Lambda d^{A}\left(x_{k}^{A}\right)\right)\left(\frac{\partial}{\partial x_{j}^{A}}, \frac{\partial}{\partial x_{i}^{A}}\right)+\sum_{j, k}^{n} g_{j} \cdot\left(d^{A}\left(p_{k}^{A}\right) \Lambda d^{A}\left(x_{k}^{A}\right)\right)\left(\frac{\partial}{\partial p_{j}^{A}}, \frac{\partial}{\partial x_{i}^{A}}\right) \\
& =\sum_{j, k}^{n} g_{j} \cdot\left[d^{A}\left(p_{k}^{A}\right)\left(\frac{\partial}{\partial p_{j}^{A}}\right) d^{A}\left(x_{k}^{A}\right)\left(\frac{\partial}{\partial x_{i}^{A}}\right)-d^{A}\left(p_{k}^{A}\right)\left(\frac{\partial}{\partial x_{i}^{A}}\right) d^{A}\left(x_{k}^{A}\right)\left(\frac{\partial}{\partial p_{i}^{A}}\right)\right] \\
& =\sum_{j, k}^{n} g_{j} \cdot\left(\delta_{k j} \cdot \delta_{k i}\right)=g_{j},
\end{aligned}
$$

and

$$
\begin{aligned}
\Omega^{A}\left(X_{H}, \frac{\partial}{\partial p_{i}^{A}}\right) & =\sum_{j=1}^{n} f_{i} \cdot \Omega^{A}\left(\frac{\partial}{\partial x_{j}^{A}}, \frac{\partial}{\partial p_{i}^{A}}\right)+\sum_{j=1}^{n} g_{j} \cdot \Omega^{A}\left(\frac{\partial}{\partial p_{j}^{A}}, \frac{\partial}{\partial p_{i}^{A}}\right) \\
& =\sum_{j=1}^{n} f_{i} \cdot \Omega^{A}\left(\frac{\partial}{\partial x_{j}^{A}}, \frac{\partial}{\partial p_{i}^{A}}\right)=\sum_{j, k}^{n} f_{j} \cdot\left(d^{A}\left(p_{k}^{A}\right) \Lambda d^{A}\left(x_{k}^{A}\right)\right)\left(\frac{\partial}{\partial x_{j}^{A}}, \frac{\partial}{\partial p_{i}^{A}}\right) \\
& =\sum_{j, k}^{n} f_{j} \cdot\left[d^{A}\left(p_{k}^{A}\right)\left(\frac{\partial}{\partial x_{j}^{A}}\right) d^{A}\left(x_{k}^{A}\right)\left(\frac{\partial}{\partial p_{i}^{A}}\right)-d^{A}\left(p_{k}^{A}\right)\left(\frac{\partial}{\partial p_{i}^{A}}\right) d^{A}\left(x_{k}^{A}\right)\left(\frac{\partial}{\partial x_{j}^{A}}\right)\right] \\
& =\sum_{j, k}^{n} f_{j} \cdot\left(-\delta_{k j} \cdot \delta_{k i}\right)=-f_{j} .
\end{aligned}
$$

As, 


$$
\begin{aligned}
i_{X_{H}} \Omega^{A} & =\sum_{i=1}^{n} g_{i} \cdot d^{A}\left(x_{i}^{A}\right)+\sum_{i=1}^{n} f_{i} \cdot d^{A}\left(p_{i}^{A}\right)=-d H \\
& =\sum_{i=1}^{n} \frac{\partial H}{\partial x_{i}^{A}} d^{A}\left(x_{i}^{A}\right)-\sum_{i=1}^{n} \frac{\partial H}{\partial p_{i}^{A}} d^{A}\left(p_{i}^{A}\right) .
\end{aligned}
$$

Thus,

$$
\left\{\begin{array}{l}
f_{i}=\frac{\partial H}{\partial p_{i}^{A}} \\
g_{i}=-\frac{\partial H}{\partial x_{i}^{A}}
\end{array}\right.
$$

where $f_{i}, g_{i} \in C^{\infty}\left(U^{A}, A\right)$. An integral curve of $X_{H}$ is a solution the following system of ordinary equation

$$
\begin{aligned}
& \left\{\begin{array}{l}
\frac{\mathrm{d}^{A}\left(x_{i}^{A}\right)}{\mathrm{d}^{A} t}=\frac{\partial H}{\partial p_{i}^{A}} \\
\frac{\mathrm{d}^{A}\left(p_{i}^{A}\right)}{\mathrm{d}^{A} t}=-\frac{\partial H}{\partial x_{i}^{A}}
\end{array} \quad \forall i=1,2, \cdots, n .\right. \\
& X_{H}=\sum_{i=1}^{n} \frac{\partial H}{\partial p_{i}^{A}} \frac{\partial}{\partial x_{i}^{A}}-\sum_{i=1}^{n} \frac{\partial H}{\partial x_{i}^{A}} \frac{\partial}{\partial p_{i}^{A}}
\end{aligned}
$$

When $\left(x_{1}, x_{2}, \cdots, x_{2 n}\right)$ is a local system of coordinates corresponding at a chart $U$ of $M$,

$$
\left.\Omega\right|_{U}=\sum_{i=1}^{n} d x_{i} \Lambda d x_{i+n}
$$

Thus,

$$
\begin{gathered}
\left.\Omega^{A}\right|_{U^{A}}=\sum_{i=1}^{n} d^{A}\left(x_{i}^{A}\right) \Lambda d^{A}\left(x_{i+n}^{A}\right), \\
\left.X\right|_{U^{A}}=\sum_{i=1}^{n} f_{i}\left(\frac{\partial}{\partial x_{i}}\right)^{A}+\sum_{i=1}^{n} f_{i+n}\left(\frac{\partial}{\partial x_{i+n}}\right)^{A}
\end{gathered}
$$

where $f_{i}, f_{i+n} \in C^{\infty}\left(U^{A}, A\right)$ for $i=1,2, \cdots, n$. For $\varphi \in C^{\infty}\left(M^{A}, A\right)$,

$$
\begin{gathered}
x_{\varphi}=\sum_{i=1}^{n} \frac{\partial \varphi}{\partial x_{n+i}^{A}} \frac{\partial}{\partial x_{i}^{A}}-\sum_{i=1}^{n} \frac{\partial \varphi}{\partial x_{i}^{A}} \frac{\partial}{\partial x_{n+i}^{A}} . \\
i_{X_{\varphi}} \Omega^{A}=-\left[\sum_{i=1}^{n} \frac{\partial \varphi}{\partial x_{i}^{A}} d^{A}\left(x_{i}^{A}\right)+\sum_{i=1}^{n} \frac{\partial \varphi}{\partial x_{n+i}} d^{A}\left(x_{n+i}^{A}\right)\right] .
\end{gathered}
$$

As

$$
\{\varphi, \psi\}_{\Omega^{A}}=X_{\varphi}(\psi),
$$

we have

$$
\{\varphi, \psi\}_{\Omega^{A}}=\sum_{i=1}^{n} \frac{\partial \varphi}{\partial x_{n+i}^{A}} \frac{\partial \psi}{\partial x_{i}^{A}}-\sum_{i=1}^{n} \frac{\partial \varphi}{\partial x_{i}^{A}} \frac{\partial \psi}{\partial x_{n+i}^{A}} .
$$

\section{References}

[1] Weil, A. (1953) Théorie des points proches sur les variétés différentiables. Colloq. Géom. Diff. Strasbourg, 111-117.

[2] Morimoto, A. (1976) Prolongation of Connections to Bundles of Infinitely Near Points. Journal of Differential Geometry, 11, 479-498. 
[3] Okassa, E. (1986-1987) Prolongement des champs de vecteurs à des variétés des points prohes. Annales de la Faculté des Sciences de Toulouse, 3, 346-366.

[4] Bossoto, B.G.R. and Okassa, E. (2008) Champs de vecteurs et formes différentielles sur une variété des points proches. Archivum Mathematicum, Tomus, 44, 159-171.

[5] Kolár, P., Michor, P.W. and Slovak, J. (1993) Natural Operations in Differential Geometry. Springer-Verlag, Berlin. http://dx.doi.org/10.1007/978-3-662-02950-3

[6] Moukala Mahoungou, N. and Bossoto, B.G.R. (2015) Hamiltonian Vector Fields on Weil Bundles. Journal of Mathematics Research, 7, 141-148. http://dx.doi.org/10.5539/jmr.v7n3p141

[7] Laurent-Gengoux, C., Pichereau, A. and Vanhaecke, P. (2013) Poisson Structures. Grundlehren der mathematischen Wissenschaften, 347. www.springer.com/series/138

[8] Okassa, E. (2007) Algèbres de Jacobi et Algèbres de Lie-Rinehart-Jacobi. Journal of Pure and Applied Algebra, 208, 1071-1089. http://dx.doi.org/10.1016/j.jpaa.2006.05.013

[9] Moukala Mahoungou, N. and Bossoto, B. G.R. Prolongation of Poisson 2-Form on Weil Bundles.

[10] Lichnerowicz, A. (1977) Les variétés de Poisson et leurs algèbres de Lie associées. Journal of Differential Geometry, 12, 253-300.

[11] Vaisman, I. (1994) Lectures on the Geometry of Poisson Manifolds. Progress in Mathematics 118, Birkhäuser Verlag, Basel. http://dx.doi.org/10.1007/978-3-0348-8495-2

[12] Bossoto, B.G.R. and Okassa, E. (2012) A-Poisson Structures on Weil Bundles. International Journal of Contemporary Mathematical Sciences, 7, 785-803.

[13] Nkou, V.B., Bossoto, B.G.R. and Okassa, E. (2015) New Characterization of Vector Field on Weil Bundles. Theoretical Mathematics and Applications, 5, 1-17. 